\title{
Investigação epidemiológica de parasitas gastrointestinais em cães de diferentes ambientes da região central do Brasil: implicações para a saúde humana-animal- ambiental
}

\author{
Epidemiological investigation of gastrointestinal parasites in dlogs from different environments in \\ central Brazil: implications for human-animal-environmental health \\ Investigación epidemiológica de parásitos gastrointestinales en perros de diferentes ambientes en la \\ región central de Brasil: implicaciones para la salud humana-animal-ambiental
}

Andernice dos Santos Zanetti ORCID: https://orcid.org/0000-0001-7888-8183 Universidade do Estado de Mato Grosso, Brasil

E-mail: andernice.zanetti@unemat.br

Bárbara Luiza Soares da Silva ORCID: https://orcid.org/0000-0001-7554-4924 Universidade do Estado de Mato Grosso, Brasil

E-mail: babilsilva30@gmail.com

Lucas França de Barros

ORCID: https://orcid.org/0000-0001-5203-178X Universidade do Estado de Mato Grosso, Brasil

E-mail: lucasmariano.f@gmail.com

Herakles Antonio Garcia

ORCID: https://orcid.org/0000-0002-1579-2405

Universidad Central de Venezuela, Venezuela

E-mail: heraklesantonio@gmail.com

Daniel Moura de Aguiar

ORCID: https://orcid.org/0000-0001-8631-522X

Universidade Federal de Mato Grosso, Brasil

E-mail: danmoura@ufmt.br

Omar Ariel Espinosa

ORCID: https://orcid.org/0000-0002-0912-9527 Faculdade do Pantanal, Brasil

E-mail: omar.espinosa@fapan.edu.br

Antonio Francisco Malheiros

ORCID: https://orcid.org/0000-0001-8169-0557

Universidade do Estado de Mato Grosso, Brasil E-mail: malheiros@unemat.br

\begin{abstract}
Resumo
O objetivo deste estudo foi descrever a diversidade e prevalência de parasitas gastrointestinais em cães dos três biomas da região central do Brasil entre 2019 e 2020. Neste estudo analisamos amostras fecais de cães através de sedimentação espontânea e centrifugo-flutuação em sacarose. Foi calculada as porcentagens de prevalências com intervalos de confiança de $95 \%$, teste qui-quadrado para avaliar diferenças significativas e índice de Shannon para calcular a diversidade e equitabilidade das espécies. Após analisar 130 amostras fecais, a prevalência de parasita intestinais foi de $57 \%$. Helmintos foram encontrados em $84 \%$ das amostras e protozoários em $71 \%$. Não houve diferença estatística significativa entre as prevalências dos biomas estudados. Os índices de diversidade e equitabilidade de Shannon foram semelhantes nos três biomas. A proporção de coinfecção foi de $65 \%$, e infecções únicas $35 \%$. Neste estudo observamos uma alta prevalência de parasitas gastrointestinais em cães dos três biomas que compõem a região central do Brasil. O índice de diversidade e equitabilidade de parasitas gastrointestinais foi semelhante nos três biomas, revelando a presença de 12 espécies de parasitas, sendo Blastocystis sp., Giardia sp. e Entamoeba coli os protozoários mais prevalentes e entre os helmintos Ancilostomídeos, Toxocara sp. e Trichuris sp. Podemos concluir que a vigilância epidemiológica de rotina, cuidados veterinários eficazes e campanhas educacionais aprimoradas sobre zoonoses parasitárias de cães, são medidas essenciais para minimizar a contaminação ambiental, evitar a transmissão de parasitas para outros hospedeiros animais e reduzir os riscos à saúde pública nos biomas da região central do Brasil.
\end{abstract}

Palavras-chave: Protozoários; Helmintos; Biomas; Saúde única. 


\begin{abstract}
The aim of this study was to describe the diversity and prevalence of gastrointestinal parasites in dogs from the three biomes in central Brazil between 2019 to 2020. In this study, we analyzed fecal samples from dogs using spontaneous sedimentation tests and centrifugal fluctuation in sucrose. Prevalence percentages were calculated with $95 \%$ confidence intervals, chi-square test to assess significant differences and Shannon index to calculate species diversity and equitability. After analyzing 130 fecal samples, the prevalence of intestinal parasites was 57\% Helminths were found in $84 \%$ of the samples and protozoa in $71 \%$. There was no statistically significant difference between the prevalence of the studied biomes. Shannon's diversity and evenness indices were similar in the three biomes. The proportion of coinfection was $65 \%$, and single infections $35 \%$. In this study, we observed a high prevalence of gastrointestinal parasites in dogs from the three biomes that make up the central region of Brazil. The index of diversity and equitability of gastrointestinal parasites was similar in the three biomes analyzed, revealing the presence of 12 species of parasites, being Blastocystis sp., Giardia sp. and Entamoeba coli the most prevalent protozoa and among the helminths Ancilostomídeos, Toxocara sp. and Trichuris sp. We can conclude that routine epidemiological surveillance, effective veterinary care and improved educational campaigns on parasitic zoonoses in dogs, are essential measures to minimize environmental contamination, prevent the transmission of parasites to other animal hosts and reduce the risks to public health in the biomes of the central region of Brazil.
\end{abstract}

Keywords: Protozoa; Helminths; Biomes; One health.

\title{
Resumen
}

El objetivo de este estudio fue escribir la diversidad y prevalencia de parásitos gastrointestinales en perros de los tres biomas del centro de Brasil entre 2029 e 2020. Analizamos muestras fecales de perros mediante pruebas de sedimentación espontánea y fluctuación centrífuga en sacarosa. Los porcentajes de prevalencia se calcularon con intervalos de confianza del $95 \%$, prueba de chi-cuadrado para evaluar diferencias significativas e índice de Shannon para calcular la diversidad y equidad de especies. Despues de analizar 130 muestras fecales, la prevalencia de parásitos intestinales fue del $57 \%$. Se encontraron helmintos en el $84 \%$ de las muestras y protozoos en el $71 \%$. No hubo diferencia estadísticamente significativa entre la prevalencia de los biomas estudiados. Los índices de diversidad y uniformidad de Shannon fueron similares en los tres biomas. La proporción de coinfecciones fue del $65 \%$ y las infecciones únicas del $35 \%$. Observamos una alta prevalencia de parásitos gastrointestinales en perros de los tres biomas que componen la región central de Brasil. El índice de diversidad y equidad de los parásitos gastrointestinales fue similar en los tres biomas, revelando la presencia de 12 especies de parásitos, siendo Blastocystis sp., Giardia sp. y Entamoeba coli los protozoos más prevalentes y entre los helmintos Ancilostomídeos, Toxocara sp. y Trichuris sp. Podemos concluir que la vigilancia epidemiológica de rutina, la atención veterinaria eficaz y las campañas educativas mejoradas sobre zoonosis parasitarias en perros, son medidas esenciales para minimizar la contaminación ambiental, prevenir la transmisión de parásitos a otros animales hospedadores y reducir los riesgos para la salud pública en los biomas de la región central de Brasil.

Palabras clave: Protozoos; Helmintos; Biomas; Salud única.

\section{Introdução}

Os cães são um dos companheiros mais comuns dos humanos entre os animais. Eles têm uma relação próxima como animais de estimação, envolvendo principalmente o desenvolvimento emocional, a socialização e o bem-estar fisiológico dos humanos (McGlade et al., 2003). Segundo a Associação Brasileira da Indústria de Produtos para Animais de Estimação - Abinpet, de acordo com dados de 2019, no Brasil existem aproximadamente 55,1 milhões de cães (Abinpet, 2019). Com base nos dados do IBGE (Instituto Brasileiro de Geografia e Estatística), em 2019 a população estimada do Brasil foi de 210 milhões de habitantes, isto resulta em cerca de 0,3 cães para cada indivíduo. Do ponto de vista epidemiológico essa é uma informação de grande importância, pois os cães servem como reservatórios, transportadores e transmissores de vários parasitas, que são zoonóticos e representam um problema de saúde pública significativo (Schar et al., 2014; Rosales \& Malheiros, 2015). Os cães desempenham um relevante papel como hospedeiro definitivo de inúmeros parasitas, eliminando ovos de helmintos e cistos e oocistos de protozoários gastrointestinais nas fezes, o que propicia a contaminação ambiental e a possível disseminação de doenças, com a perpetuação do ciclo biológico (Ho et al., 2006; Rosales \& Malheiros, 2015).

Parasitas gastrointestinais contaminam o meio ambiente, especialmente o solo/areia, favorecendo o risco de infecções ao homem. Entre os helmintos temos os parasitas generalizados Ancylostoma caninum que causa a Larva Migrans Cutânea e enterite eosinofílica, e Toxocara canis responsável pela Larva Migrans Visceral, infecções de grande interesse para saúde porque 
geralmente resultam em doença multissistêmica por migração visceral e danos que podem afetar órgãos importantes como olhos, fígado e cérebro (McCarthy \& Moore, 2000; Diba et al., 2004). As infecções intestinais por protozoários podem causar diarreia e perda de peso e aumentam a suscetibilidade a outros patógenos (McCarthy \& Moore, 2000).

Estudos realizados no Brasil, tem apontado o cão como importante reservatório de zoonoses enteroparasitárias. A soroprevalência para infecção por Toxocara sp. varia de 3 a 15\% em diferentes regiões (Nunes et al., 2018; Zanetti et al., 2019). Já a soroprevalência para infecção por Ancilostomídeos é mais elevada e varia de 25 a 47\% (Curi et al., 2017; Nunes et al., 2018). A alta prevalência de infecções parasitárias intestinais nesses animais, associada a fatores ambientais favoráveis e ao comportamento humano, levando a práticas inapropriadas de convívio, além da falta de manejo adequado dos cães domésticos, incluindo a ausência de vacinas, desparasitação e controle populacional, aumentam os riscos de transmissão zoonótica.

Em áreas com alta biodiversidade, como o Brasil, os cães domésticos têm sido frequentemente implicados como fonte de infecção para animais silvestres (Sevá et al., 2018). Além disso, o efeito contrário, relacionado à transferência de infecções parasitárias de animais silvestres para cães domésticos, pode ocorrer, que por sua vez transmitem a humanos dentro ou fora de seus habitats naturais, nesse caso, a prevalência de parasitas caninos representa um bioindicador de contaminação ambiental.

Devido à crescente proximidade entre o homem, o cão e o ambiente, é cada vez mais forte e presente o conceito de saúde única (One Health) que contribui para a promoção integrada da saúde humana, animal e do meio ambiente (Paul \& Gibbs, 2014), portanto a intensificação da vigilância epidemiológica, a atenção veterinária e a educação sanitária e ambiental para a população, são algumas das estratégias para o efetivo controle e prevenção de doenças promovendo a saúde humana, animal e ambiental. Por este motivo, o objetivo desse estudo foi descrever a diversidade e prevalência de parasitas gastrointestinais zoonóticos e não zoonóticos em cães dos três biomas da região central do Brasil.

\section{Metodologia}

\subsection{Local do estudo e coletas das amostras}

Este estudo faz parte de um projeto mais amplo de pesquisa sobre 'Diversidade e caracterização molecular de enteroparasitas de diferentes hospedeiros de três biomas brasileiros' (Projeto Universal CNPq 423391/2018-6).

Trata-se de um estudo exploratório, descritivo e transversal com abordagem quantitativa (Pereira et al., 2018), realizado com amostras de fezes de cães dos três biomas presentes no estado de Mato Grosso. Os municípios dos locais de coleta que compõem os biomas foram: Pantanal (Cáceres), Cerrado (Tangará da Serra) e Amazônia (Alta Floresta). A coleta de amostras foi realizada no período de abril de 2019 a fevereiro de 2020.

As amostras dos cães foram coletadas por busca ativa diretamente do ambiente, por meio da espera, observação e coleta das fezes frescas, sem a necessidade de anestesiar, sacrificar ou manusear os animais.

\subsection{Procedimentos laboratoriais}

As fezes coletadas foram encaminhadas ao Laboratório de Biologia Parasitária da Universidade do Estado de Mato Grosso. Cada amostra foi submetida às seguintes técnicas parasitológicas: sedimentação espontânea de acordo com Hoffmann et al. (1934) e centrifugo-flutuação em sacarose (Sheather, 1923). A identificação de helmintos e protozoários foi realizada morfologicamente por comparação com registros publicados. As amostras foram consideradas positivas quando pelo menos um estágio do ciclo de vida de um parasita, um ovo ou larva de helminto e/ ou cistos ou oocistos de protozoários, foram detectados em pelo menos uma das técnicas utilizadas. 
Todos os ovos / cistos dos parasitas foram identificados em nível de gênero e / ou espécie, sempre que possível, exceto os Ancilostomídeos onde os gêneros não podem ser distinguidos para todas as infecções (por exemplo, Ancylostoma e Uncinaria).

\subsection{Análises estatísticas}

Dados parasitológicos foram analisados para estimar a prevalência e diversidade de espécies de parasitas detectados em geral e dentro de cada bioma amostrado. Os dados foram digitados em uma planilha do Excel e transferidos para o Stata versão 13.1 (Stata Corp; College Station, Estados Unidos da América). Estatística descritiva foi utilizada calculando as porcentagens de prevalências com intervalos de confiança de $95 \%$. Foi calculado o teste do qui-quadrado para avaliar se houve uma diferença significativa entre os biomas estudados, com valor $p<0,05$ interpretado como estatisticamente significativo. $\mathrm{O}$ índice de diversidade de Shannon foi utilizado para calcular a diversidade, abundância e equitabilidade das espécies de parasitas presentes nas amostras de cães dos três biomas.

\section{Resultados}

No total foram analisadas 130 amostras fecais de cães dos três biomas. Em relação à distribuição das amostras analisadas segundo o bioma estudado, foram coletadas no Pantanal 38,6\% (50) das amostras e no Cerrado e Amazônia, ambos com 30,7\% (40) (Tabela 1).

\subsection{Prevalência geral dos parasitas gastrointestinais na população de cães amostrada}

A prevalência geral de infecção por parasitas intestinais nas 130 amostras analisadas foi de $57 \%$ ( 75 amostras). Infecções por protozoários foram encontradas em 71\% (53) das amostras positivas, sendo Blastocystis sp. o mais prevalente (52\%), seguido de Giardia sp. e Entamoeba coli, ambos com 12\% (Tabela 1).

Os helmintos foram encontrados em 84\% (63) das amostras positivas. Os Ancilostomídeos foram os mais prevalentes (64\%), seguido de Toxocara sp. (31\%) e Trichuris sp. (11\%). A porcentagem de prevalência de todos parasitas encontrados está apresentada na Tabela 1. 
Tabela 1. Porcentagem de prevalência (95\% de IC - Intervalo de Confiança entre parênteses) dos parasitas gastrointestinais identificados em amostras de 130 cães.

\begin{tabular}{|c|c|c|c|c|}
\hline & $\begin{array}{c}\text { Cáceres - } \\
\text { Pantanal } \\
(\mathrm{N}=50) \\
\%(\text { IC } 95 \%) \\
\end{array}$ & $\begin{array}{c}\text { Tangará da Serra } \\
\text { - Cerrado } \\
(\mathrm{N}=40) \\
\%(\text { IC } 95 \%) \\
\end{array}$ & $\begin{array}{l}\text { Alta Floresta - } \\
\text { Amazônia } \\
(\mathrm{N}=40) \\
\%(\text { IC 95\%) }\end{array}$ & $\begin{array}{l}\text { Total das } \\
\text { amostras } \\
(\mathrm{N}=130) \\
\%(\mathrm{IC} 95 \%) \\
\end{array}$ \\
\hline Prevalência total & $68(54-79)$ & $47(33-63)$ & $55(40-69)$ & $57(45-70)$ \\
\hline \multicolumn{5}{|c|}{ Helmintos } \\
\hline Ancilostomídeos ** & $74(57-85)$ & $47(27-68)$ & $64(43-80)$ & $64(53-74)$ \\
\hline Toxocara sp.** & $35(21-52)$ & $21(9-43)$ & $32(16-53)$ & $31(21-42)$ \\
\hline Trichuris sp. ** & $9(3-23)$ & $11(3-31)$ & $13(3-32)$ & $11(9-23)$ \\
\hline Ascaris sp. $* *$ & $6(2-19)$ & $5(1-25)$ & $9(3-28)$ & $7(3-15)$ \\
\hline Strongyloides sp. $* *$ & $0(0)$ & $0(0)$ & $5(1-22)$ & $1(0-7)$ \\
\hline \multicolumn{5}{|c|}{ Protozoários } \\
\hline Blastocystis sp. ** & $62(45-76)$ & $58(36-77)$ & $32(16-53)$ & $52(41-63)$ \\
\hline Giardia sp. $* *$ & $18(8-34)$ & $5(1-25)$ & $9(3-28)$ & $12(6-21)$ \\
\hline Entamoeba coli & $9(3-23)$ & $11(3-31)$ & $18(7-39)$ & $12(6-21)$ \\
\hline Chilomastix sp. & $6(2-19)$ & $0(0)$ & $0(0)$ & $3(1-9)$ \\
\hline Complexo E. histolytica/dispar ** & $6(2-19)$ & $11(3-31)$ & $0(0)$ & $5(2-13)$ \\
\hline Eimeria sp. & $6(2-19)$ & $11(3-31)$ & $0(0)$ & $5(2-13)$ \\
\hline Neospora sp. & $0(0)$ & $0(0)$ & $9(3-28)$ & $3(1-9)$ \\
\hline
\end{tabular}

Fonte: Autores (2021).

Na Tabela 1 é possível observar os helmintos e protozoários com potencial zoonótico identificados nas amostras dos cães coletados nos três biomas, com suas porcentagens de prevalência e intervalo de confiança IC de 95\%. As prevalências de infecções por bioma bem como do total de amostras analisadas, foram altas.

\subsection{Prevalência da infecção por parasitas gastrointestinais segundo o bioma estudado}

Entre as amostras positivas de cada bioma estudado, o Pantanal apresentou uma prevalência de infecção parasitária de 68\% (34 amostras), seguido da Amazônia em 55\% (22) e o Cerrado com 47\% (19). Após aplicar o teste de qui-quadrado observamos que não houve diferença significativa entre as prevalências dos três biomas (dados não apresentados).

A prevalência da infecção por protozoários intestinais em cada bioma estudado, Pantanal, Amazônia e Cerrado, foi de $73 \%, 84 \%$ e 54\%, respectivamente. Em relação à prevalência da infecção por espécies de helmintos encontrada nestes três biomas foi de $88 \%, 68 \%$ e $91 \%$ respectivamente.

Dentre os protozoários, Blastocystis sp. foi a espécie mais prevalente nos três biomas, enquanto que dos helmintos foram os Ancilostomídeos. A distribuição das espécies e prevalências dos parasitas intestinais encontrados nos biomas estudados pode ser observada na Figura 1. 
Figura 1. Distribuição das espécies e prevalência dos parasitas gastrointestinais encontrados em cães nos biomas estudados.

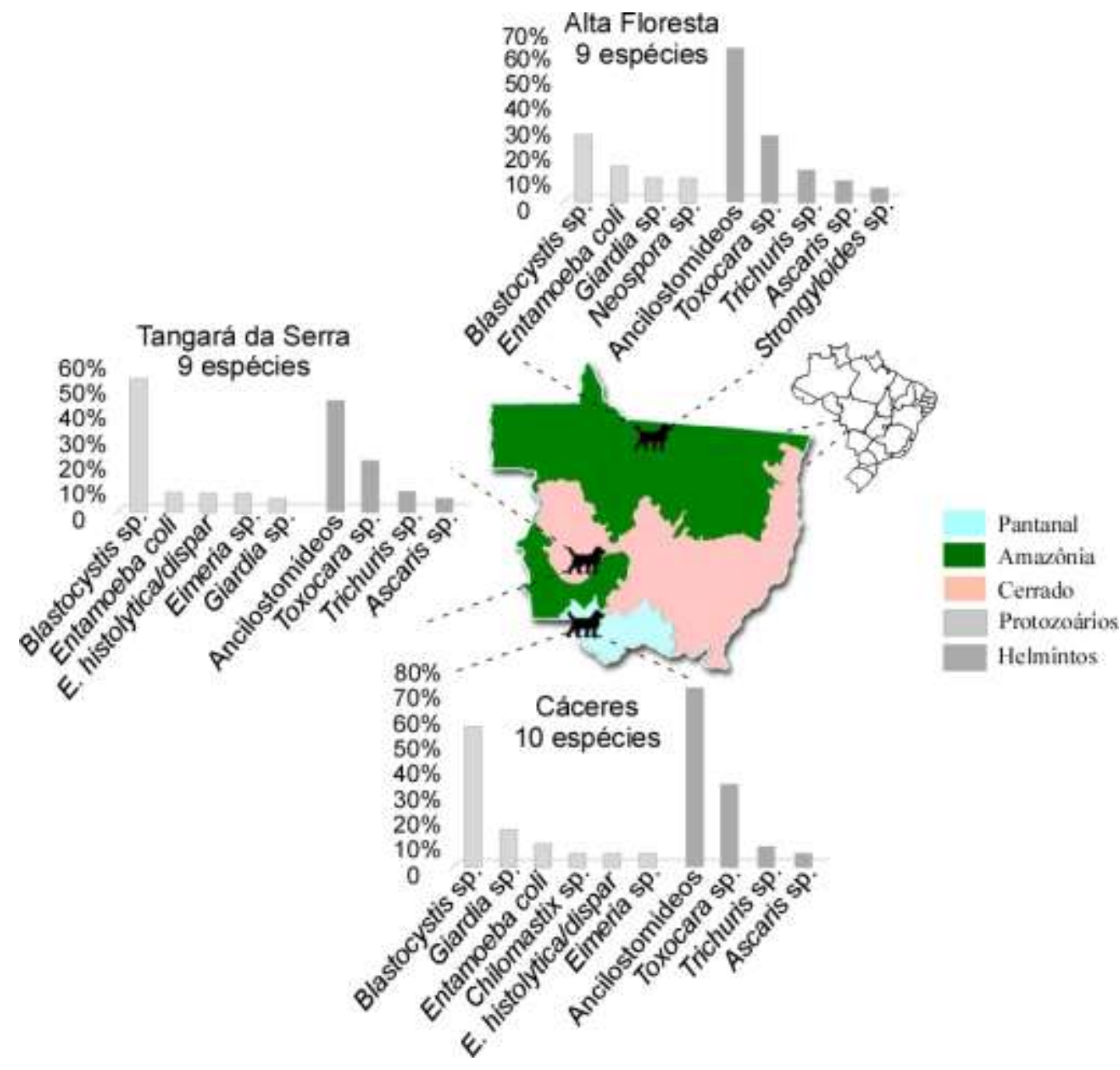

Fonte: Autores (2021).

A Figura 1 ilustra a localização das cidades nos respectivos biomas que ocorreram as coletas das 130 amostras de cães. Apresenta o quantitativo das diferentes espécies de parasitas intestinais encontrados com 10 espécies no Pantanal e 9 no Cerrado e Amazônia.

Esses resultados foram comparados com estudos de prevalência em cães publicados nos diferentes biomas brasileiros (Tabela 2). 
Tabela 2. Prevalência (\%) dos parasitas gastrointestinais mais comuns obtidos nesse estudo e de estudos similares nos diferentes biomas brasileiros.

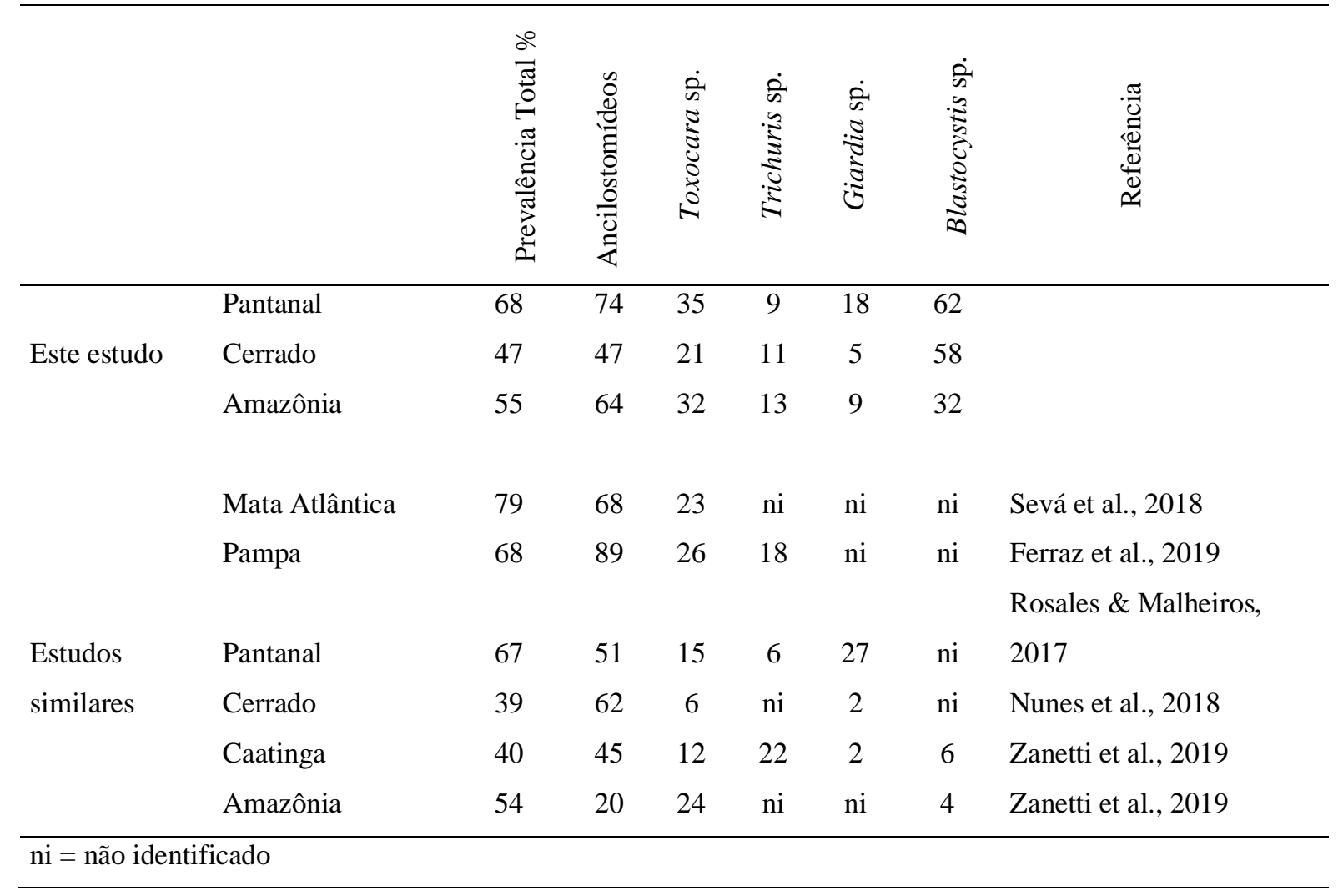

Fonte: Autores (2021).

Na Tabela 2 observamos as prevalências dos principais parasitas intestinais encontrados em nosso estudo, nos três biomas, e comparamos com as prevalências encontradas nos demais biomas brasileiros relatadas em outros estudos similares.

\subsection{Diversidade e equitabilidade das espécies de parasitas intestinais}

Um total de 12 espécies de parasitas gastrointestinais foram encontrados nos três biomas estudados. No Pantanal, os cães infectados apresentaram 83\% das espécies encontradas (10/12). Na Amazônia e no Cerrado, os cães infectados apresentaram ambos 75\% (9/12) das espécies encontradas (Figura 1).

A diversidade de espécies, refletida no índice de diversidade e equitabilidade de Shannon, foi semelhante em todos biomas (Tabela 3). 
Tabela 3. Índice de diversidade e equitabilidade de Shannon dos parasitas gastrointestinais de cães encontrados entre os diferentes biomas analisados.

\begin{tabular}{|c|c|c|c|}
\hline Bioma & Tipo parasitário & Diversidade & Equitabilidade \\
\hline & Protozoários & 0,9577 & 0,6607 \\
\hline Pantanal & Helmintos & 0,8719 & 0,8421 \\
\hline \multicolumn{2}{|c|}{ Pantanal Total } & 1,8296 & 0,7362 \\
\hline & Protozoários & 0,9687 & 0,6683 \\
\hline Cerrado & Helmintos & 0,8739 & 0,8441 \\
\hline \multicolumn{2}{|c|}{ Cerrado Total } & 1,8427 & 0,7415 \\
\hline & Protozoários & 0,7715 & 0,5322 \\
\hline Amazônia & Helmintos & 1,0713 & 1,0347 \\
\hline \multicolumn{2}{|c|}{ Amazônia Total } & 1,8429 & 0,7416 \\
\hline
\end{tabular}

Fonte: Autores (2021).

Na Tabela 3 é possível observar que nos biomas Pantanal e Cerrado, os protozoários apresentam um maior valor do índice de Shannon (diversidade) e uma alta distribuição equitativa quando comparados com os helmintos. Em relação à Amazônia, os helmintos apresentaram maior índice de Shannon e alta distribuição equitativa quando comparado com os protozoários.

\subsection{Prevalência de Coinfecções}

Quando analisadas as amostras positivas segundo o número de espécies encontradas, observamos que a prevalência das infecções simples foi de 35\% (26/75), e o restante 65\% (49/75), corresponderam à prevalência de infeções mistas.

Em relação à coinfecção, as infecções duplas foram as mais prevalentes (53\%) seguida de infecções triplas (37\%), quádruplas (6\%) e quíntuplas (4\%). Das infecções duplas a associação entre Blastocystis sp. + Ancilostomídeos, foram as mais comuns (oito amostras), seguida da associação entre Toxocara sp. + Ancilostomídeos (quatro), Blastocystis sp. + Toxocara sp. (duas) e Blastocystis sp. + Ascaris sp., Giardia sp. + Ancilostomídeos, Giardia sp. + Trichuris sp., Eimeria sp. + Ancilostomídeos, Entamoeba coli + Trichuris sp., Eimeria sp. + Entamoeba histolytica/díspar, Neospora sp. + Ancilostomídeo, Trichuris sp. + Toxocara sp., Ancilostomídeos + Strongyloides sp., Neospora sp. + Toxocara sp., Entamoeba coli + Ancilostomídeos, Toxocara sp. + Ascaris sp., todos com uma amostra.

Dentre as infecções triplas, a associação entre Blatocystis sp. + Ancilostomídeos + Toxocara sp., foram as mais comuns (seis amostras), seguida de Blastocystis sp. + Entamoeba coli + Ancilostomídeos (três), Blastocystis sp. + Giardia sp. + Ancilostomídeos (duas) e Giardia sp. + Ancilostomídeos + Toxocara sp., Blastocystis sp. + Chilomastix sp. + Toxocara sp., Blastocystis sp. + Entamoeba histolytica/dispar + Trichuris sp., Blastocystis sp. + Entamoeba histolytica/dispar + Toxocara sp., Blastocystis sp. + Trichuris sp. + Ancilostomídeos, Eimeria sp. + Ancilostomídeos + Ascaris sp., Entamoeba coli + Trichuris sp. + Ancilostomídeos, todos com uma amostra cada. Dentre as infecções quádruplas e quíntuplas, todas associações estavam presentes em uma amostra, Blastocystis sp. + Entamoeba coli + Giardia sp. + Ancilostomídeos, Blastocystis sp. + Entamoeba histolytica/dispar + Ancilostomídeos + Trichuris sp., Blastocystis sp. + Giardia sp. + Ancilostomídeos + Ascaris sp., Blastocystis sp. + Giardia sp. + Entamoeba coli + Ancilostomídos + Toxocara sp. e Blastocystis sp. + Eimeria sp. + Ancilostomídeos + Trichuris sp. + Ascaris sp. 


\section{Discussão}

A taxa de prevalência de parasitas intestinais nos cães das regiões pesquisadas em nosso estudo (57\%) foi elevada, coincidindo com a média encontrada em outras regiões do Brasil, a qual varia entre 39\% e 79\% (Nunes et al., 2018; Sevá et al., 2018).

A infecção por helmintos intestinais (84\%) foi maior que as encontradas por protozoários (71\%). Os helmintos mais prevalentes neste estudo foram Ancilostomídeos (64\%), Toxocara sp. (31\%) e Trichuris sp. (11\%). Por outro lado, os protozoários mais encontrados foram Blastocystis sp. (52\%\%) e Giardia sp. e Entamoeba coli, ambos com 12\%. Contudo, a maioria das espécies de parasitas identificados em nosso trabalho possui potencial zoonótico e distribuição mundial (Stensvold et al., 2007; Bolfa et al., 2019; Maganga et al., 2019).

Quando analisados os três biomas, observamos semelhanças na prevalência e nos tipos de parasitas gastrointestinais identificados. Embora as prevalências sejam diferentes em cada bioma, Pantanal (68\%), Amazônia (55\%) e Cerrado (47\%), não houve diferença estatística significativa entre eles. A semelhança nas prevalências encontradas nos três biomas estudados, pode ser explicada devido as temperaturas e condições de umidade, o manejo dos proprietários e programas sanitários no controle de agentes infecciosos ser praticamente os mesmos nos três municípios a que eles pertencem.

As prevalências gerais de parasitas gastrointestinais nos três biomas detectadas neste estudo, foram semelhantes às relatadas em outros estudos conduzidos nos mesmos biomas (Rosales \& Malheiros, 2017; Nunes et al., 2018; Zanetti et al., 2019). Porém, quando analisado a prevalência por espécie de parasita, este estudo detectou prevalências mais altas dos principais parasitas gastrointestinais, exceto Giardia sp. no Pantanal e Ancilostomídeos no Cerrado, que relataram uma taxa mais alta de infecção do que a encontrada em nossa pesquisa (Rosales \& Malheiros, 2017; Zanetti et al., 2019).

A diversidade de espécies foi alta em todos os biomas, já que foram encontradas 12 espécies de parasitas no total, sendo sete protozoários e cinco helmintos. Quando segregadas as espécies por biomas, observamos que no Pantanal foram encontradas 10 espécies e na Amazônia e Cerrado, nove espécies cada. A análise de diversidade e equitabilidade de Shannon registrou índices similares, portanto, todos os biomas apresentaram uma diversidade considerável de espécies e uma distribuição equitativa de parasitas gastrointestinais.

A alta e disseminada prevalência de parasitas gastrointestinais, principalmente Ancilostomídeos e Toxocara sp., revela a falta de controle preventivo das populações caninas, e que o risco de doenças zoonóticas em cães está fortemente presente nas áreas de estudo. Esses resultados podem estar relacionados com o desconhecimento dos proprietários de cães sobre o risco de adquirir zoonoses, na ausência de medidas profiláticas, falta de programas governamentais relacionados à saúde e zoonoses e na alta contaminação ambiental devido ao comportamento livre dos cães e práticas inadequadas de higiene e cuidados com os animais, levando ao acúmulo de fezes no solo e disseminação de ovos de parasitas (Balassiano et al., 2009). O solo contaminado é uma importante fonte de infecções por parasitas gastrointestinais, principalmente por Toxocara sp., Ascaris sp., Trichuris sp. e Ancilostomídeos (Tiyo et al., 2008), todos identificados em nosso estudo com prevalências significativas.

Os parasitas gastrointestinais caninos mais prevalentes encontrados em nosso estudo (Ancilostomídeos, Toxocara sp. e Trichuris sp.) não diferiram de outros estudos similares no Brasil (Rosales \& Malheiros, 2017; Nunes et al., 2018; Sevá et al., 2018; Ferraz et al., 2019; Zanetti et al., 2019). No entanto, outros parasitas intestinais menos comuns também foram encontrados, provavelmente devido à proximidade com outras espécies hospedeiras de animais domésticos, como é o caso de Eimeria sp. e Ascaris sp., que são gêneros típicos de galinhas e porcos (Vicente et al., 1997). Essas infecções podem acontecer de forma acidental, uma vez que cães frequentemente ingerem vísceras galinha e porcos ou alimentos contaminados com fezes destes animais. No entanto, como os cães estão implicados na transmissão de Ancilostomídeos e Ascaris sp. (entre outros parasitas), mesmo aqueles menos prevalentes, para os humanos, merecem maior atenção. 
Nossos resultados no geral, demonstraram que a coinfecção com mais de um parasita gastrointestinal foi maior que as infecções únicas nos cães dos três biomas analisados. A infecção do hospedeiro por uma espécie de parasita pode alterar a suscetibilidade do hospedeiro a outras espécies parasitárias (Viney \& Graham, 2013), portanto, o fato do ambiente ter níveis elevados de contaminação enteroparasitária pode aumentar a probabilidade de coinfecção. O papel da infecção simultânea por múltiplos parasitas na saúde do hospedeiro geralmente não é bem estudado, porém as coinfecções estão associadas a maiores efeitos negativos à saúde (Donohue et al., 2019). A coinfecção foi mais prevalente em nossos resultados, portanto, deve ser um foco importante para investigações da contaminação ambiental.

\section{Conclusão}

Neste estudo observamos uma alta prevalência de parasitas gastrointestinais em cães dos três biomas que compõem a região central do Brasil. O índice de diversidade e equitabilidade de parasitas gastrointestinais foi semelhante nos três biomas analisados, revelando a presença de 12 espécies de parasitas, sendo Blastocystis sp., Giardia sp. e Entamoeba coli os protozoários mais prevalentes, e Ancilostomídeos, Toxocara sp. e Trichuris sp. os helmintos de maior prevalência. Os resultados mostram que os cães podem ser fonte de infecção para o homem e favorecer a contaminação ambiental e o estabelecimento de novos casos, já que vários parasitas identificados possuem elevado potencial zoonótico, com risco para a saúde dos animais e humana.

A partir dos nossos resultados, podemos concluir que a vigilância epidemiológica de rotina, cuidados veterinários eficazes e campanhas educacionais aprimoradas sobre zoonoses parasitárias de cães, são medidas essenciais para minimizar a contaminação ambiental, evitar a transmissão de parasitas para outros hospedeiros animais e reduzir os riscos à saúde pública nos biomas da região central do Brasil e outras áreas com condições climáticas semelhantes.

Uma avaliação mais aprofundada das espécies de parasitas intestinais identificadas através de análise molecular permitirá examinar a presença de potencial zoonótico nessas regiões, assim, futuras pesquisas científicas que visem a ocorrência e distribuição de parasitas intestinais em cães nas diferentes regiões brasileiras, contribuirão com a vigilância epidemiológica e facilitarão a compreensão das correlações entre as doenças zoonóticas em cães e as populações mais susceptíveis.

\section{Agradecimentos}

Os autores agradecem o apoio financeiro do Conselho Nacional de Desenvolvimento Científico e Tecnológico - CNPq (Projeto Universal 423391/2018-6) e à Universidade do Estado de Mato Grosso - UNEMAT com uma bolsa de estudo patrocinada por esta instituição. D.M. Aguiar é bolsista de pesquisa do CNPq (bolsa no: 303677 / 2018-0).

\section{Referências}

Abinpet (2019). Associação Brasileira da Indústria de Produtos para Animais de Estimação. http://abinpet.org.br/site/mercado/.

Balassiano, B. C. C., Campos, M. R., Menezes, R. D. C. A. A., \& Pereira, M. J. S. (2009). Factors associated with gastrointestinal parasite infection in dogs in Rio de Janeiro, Brazil. Preventive Veterinary Medicine, 91(2), 234-240.

Bolfa, P., Callana, J. J., Ketzis, J., Marchi, S., Cheng, T., Huynh, H., Lavinder, T., Boey, K., Hamilton, C., \& Kelly, P. (2019). Infections and pathology of freeroaming backyard chickens on St. Kitts, West Indies. Journal of Veterinary Diagnostic Investigation, 1(1), 1-7.

Curi, N. H. A, Paschoal, A. M. O., Massara, R. L., Santos, H. A., Guimarães, M. P., Passamani, M., Chiarello, A. G. (2017). Risk factors for gastrointestinal parasite infections of dogs living around protected areas of the Atlantic Forest: implications for human and wildlife health. Brazilian Journal Biology, 77(2), $388-395$.

Diba, V. C., Whitty, C. J. M., \& Green, T. (2004). Cutaneous larva migrans acquired in Britain. Clinical and experimental dermatology, 29(5), 555-556.

Donohue, R. E., Cross, Z. K., \& Michael, E. (2019). The extent, nature, and pathogenic consequences of helminth polyparasitism in humans: A meta- analysis. PLoS Neglected Tropical Disease, 13(6), e0007455. 
Ferraz, A., Pires, B. S., Santos, E. M., Evaristo, T. A., Leão, M. S., Mello, C. C. S., Castro, T. A., Pinto, D. M., \& Nizoli, L. Q. (2019). Ocorrência de parasitos gastrintestinais de cães, com potencial zoonótico no entorno de escolas municipais de educação infantil em Pelotas, RS, Brasil. Revista de Ciências Veterinárias e Saúde Pública, 6(2), 291-301.

Ho, S., Watanabe, Y., Lee, Y., Shih, T., Tu, W., \& Ooi, H. Survey of gastrointestinal parasitic infections in quarantined dogs in Taiwan. The Journal of Veterinary Medical Science, 68(1), 69-70.

Hoffman, W. A., Pons, J. A., \& Janner, J. L. (1934). The sedimentation concentration method in Schistosomiasis mansoni. Journal of Public Health Tropical Medicine, 9(1) 283-291.

Maganga, G. D., Kombila, L. B., Boundenga, L., Kinga, I. C. M., Obame-Nkoghe, J., Tchoffo, H., Tchoffo, H., Gbati, O. B., \& Awah-Ndukum, J. (2019). Diversity and prevalence of gastrointestinal parasites in farmed pigs in Southeast Gabon, Central Africa. Veterinary World, 12(12), $1888-1896$.

Mccarthy, J. \& Moore, T.A. (2000). Emerging helminth zoonoses. International Journal for Parasitology, 30(12-13), 1351-1359.

McGlade, T. R., Robertson, I. D., Elliot, A. D., Read, C., \& Thompson, R. C. (2003). Gastrointestinal parasites of domestic cats in Perth, Western Australia. Veterinary Parasitology, 117(4), 251-262.

Nunes, H. C., Moura, A. S., Gontijo, E. E. L., \& Silva, M. G. (2018). Prevalência de parasitas intestinais em cães triados no Centro de Controle de Zoonoses de Gurupi, Tocantins. Revista Cereus, 10(3), 25-31.

Paul, E. \& Gibbs, J. (2014). The Evolution of One Health: a decade of progress and challenges from the future. Veterinary Record, $174(4), 21-29$.

Pereira, A. S., Shitsuka, D. M., Parreira, F. J., Shitsuka, R. (2018). Metodologia da pesquisa científica. UAB/NTE/UFSM. https://repositorio.ufsm.br/bitstream/handle/1/15824/Lic_Computacao_Metodologia-Pesquisa-Cientifica.pdf?sequence=1

Rosales, T. F. L. \& Malheiros, A. F. (2017). Contaminação Ambiental por enteroparasitas presentes em fezes de cães em uma região do Pantanal. $O$ Mundo da Saúde, 41(3), 368-377.

Schar, F., Inpankaew, T., Traub, R. J., Khieu, V., Dalsgaard, A., Chimnoi, W., Chhoun, C., Sok, D., Marti, H., Muth, S., \& Odermatt, P. (2014). The prevalence and diversity of intestinal parasitic infections in humans and domestic animals in a rural Cambodian village. Parasitology International, 63(4), 597-603.

Sevá, A. P., Pena, H. F. J., Nava, A., Sousa, A. O., Holsback, L., \& Soares, R. M. (2018). Endoparasites in domestic animals surrounding an Atlantic Forest remnant, in São Paulo State, Brazil. Brazilian Journal of Veterinary Parasitology, 27(4), 12-18.

Sheather, A. L. (1923). The detection of intestinal protozoa and mange parasites by a flotation technique. Journal of Comparative Pathology, 36(3), 266-275.

Stensvold, C. R., Suresh, G. K., Tan, K. S., Thompson, R. C., Traub, R. J., Viscogliosi, E., Yoshikawa, H., \& Clark, C. G. (2007). Terminology for Blastocystis of subtype a consensus. Trends of Parasitology, 23(3), 93-96.

Vicente, J. J., Rodrigues, H. D. O., Gomes, D. C., \& Pinto, R. M. (1997). Brazilian nematodes. Part V: nematodes of mammals. Revista Brasileira de Zoologia, $14(1), 1-452$.

Viney, M. E. \& Graham, A. L. (2013). Patterns and Processes in Parasite Co-Infection. Advences in Parasitology, 82(2), 321-69.

Tiyo, R., Guedes, T. A., Falavigna, D. L. M, \& Falavigna-Guilherme, A. L. (2008). Seasonal contamination of public squares and lawns by parasites with zoonotic potential in southern Brazil. Journal of Helminthology, 82(1), 1-6.

Zanetti, A. S., Silva Junior, I. C., Barros, L. F., Domínguez, O. A. E., Lima, G. S., Silva, A. S., Danelichen, P. S., Silva, S. L., Moreira, L. M., Shaw, J. J., \& Malheiros, A. F. (2019). Parasitas intestinais em cães provenientes dos biomas do nordeste brasileiro: aspecto zoonótico e ambiental. Revista Ibero Americana de Ciências Ambientais, 10(3), 42-51. 